\title{
Effect of friction on the force distribution in sheared granular materials
}

\author{
A. Singh, V. Magnanimo \& S. Luding \\ Multi Scale Mechanics (MSM), Faculty of Engineering Technology, MESA+, \\ University of Twente, P.O. Box 217, 7500 AE Enschede, The Netherlands.
}

\begin{abstract}
The effect of friction on the quasi-static behavior of dense granular media is the subject of this work. The system is studied in a split-bottom ring shear cell, using Discrete Element Method simulations (DEM). When shear stress and deviatoric fabric are analyzed locally as functions of pressure and shear rate, they are found to increase with contact friction; saturation is reached for high friction with the strong contact network dominating. We start from this observation and look at the probability distribution functions (PDFs) of both normal and tangential forces. They behave in a similar fashion, i.e., the probability of weak forces and heterogeneity increase with friction. On the other hand, we find that the difference between the two directions (anisotropy) increases with friction in the case of normal forces, whereas it stays almost unaffected for tangential forces.
\end{abstract}

\section{Introduction}

What do sand, rice, coffee powders and cocoa powder have in common? They all are granular materials: a collection of non-Brownian, macroscopic particles with dissipative interactions. Their intrinsic nature leads to great deal of interesting phenomena like segregation, jamming, clustering, arching and shear-band formation (Jaeger et al. 1996). It is now well established that the microstructure of granular matter plays a significant role in the overall constitutive behavior. Finding a connection between the continuum response and kinematics at microscale is a great challenge and involves the so-called micromacro transition (Lätzel et al. 2000). Particle simulations are relatively recent powerful tools that allow to track individual particles with complex interaction by solving Newton's laws of motion. The micromacro transition is often applied on small, seemingly homogeneous representative volume elements (RVE)s. An alternative is to use an inhomogeneous geometry where static and dynamic, flowing zones i.e. high density and dilated zones coexist - at various confining pressure levels. In these kind of systems, by local averaging over adequate representative volume elements (RVE)s inside which all particles are assumed to behave similarly, one can obtain local continuum relations covering a wide range of system states. Such a procedure has been performed systematically in two-dimensional Couette ring shear cell (Lätzel et al. 2000), and three dimensional split-bottom ring shear cell (Luding 2008b, Luding and Alonso-Marroquín 2011). One special property of this set-up is the fact that a wide, stable shear band initiates at the bottom split and remains far away from side walls. The free surface of the split-bottom shear cell allows to scan a wide range of confining pressure, due to the weight of the material and determined by the filling height. Constitutive relations involving densities, pressures and shear-rates can be obtained from a single simulation by performing local and temporal averaging in the steady state. Simulations of dry particles with and without friction have been validated with experiments. A quantitative agreement was found with deviations as small being $10 \%$ as reported in (Luding 2008b, Luding 2008a).

Under shear, the microstructure of a grain packing develops anisotropic features in both stress and contact network. The anisotropy depends on preparation history of the material and at the same time on the contact and particle properties such as stiffness, roughness and angularity of particles. The effect of particle friction on macroscopic quantities such as shear strength has been the focus of our previous studies (Luding 2008b, Singh et al. 2013). In this paper, we focus on the problem at microlevel and study the effect of particle friction on the steady state behavior by investigating the probability distribution of forces along the eigen-directions of the local strain rate tensors.

The paper is organized in 4 main parts. Section 2 describes the model system specifying the geometry, particle properties and interaction laws. In section 3, the results from samples with different inter-particles friction on the force distribution are presented. Finally, 4 is the section dedicated to discussion of results.

\section{Model System Geometry}

In this section, the methodology of our molecular dynamics types simulations is briefly discussed. The details about particle properties are presented in Sec. 2.1 and we show our numerical setup in Sec. 2.2. In Sec. 2.3, we briefly discuss our averaging methodology. 


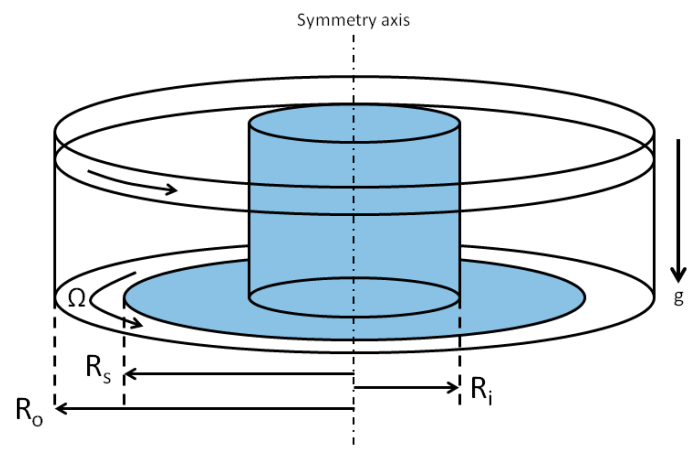

Figure 1: A sketch of our numerical setup consisting of a fixed inner part (light blue shade) and a rotating outer part (white). The white part of the base and the outer cylinder rotate with the same angular velocity $\Omega$ around the symmetry axis. The inner, split, and outer radii are given by $R_{i}=0.0147 \mathrm{~m}, R_{s}=0.085 \mathrm{~m}$, and $R_{O}=0.11 \mathrm{~m}$, respectively, where each radius is measured from the symmetry axis. The gravity $g$ points downwards as shown by arrow.

\subsection{Numerical simulations}

Discrete Element Methods (DEM), provide numerical solutions of Newton's equations of motion based on the specification of particle properties viz. stiffness, density, radius and an interaction law like Hertzian/Hookean (Cundall 1971). Simulation methodology and material parameters used in this study are the same as in our previous work (Luding 2008b). The normal contact force is related to overlap through a linear contact law, $f_{n}=k_{n} \delta_{n}$, with stiffness $k_{n}$, if $\delta \geq 0$. In tangential direction, the force $f_{t}=k_{t} \delta_{t}$ is also proportional to the tangential displacement of the contact point, with a stiffness $k_{t}$. The tangential force is limited by Coulombs law for sliding $f_{t} \leq \mu_{p} f_{n}$, i.e. for $\mu_{p}=0$ tangential forces do not exist. For more details on the contact model see (Luding 2008b) and references therein.

The system is filled with $N \approx 37000$ spherical particles with density $\rho=2000 \mathrm{~kg} / \mathrm{m}^{3}=2 \mathrm{~g} / \mathrm{cm}^{3}$. The average size of particles is $a_{0}=1.1 \mathrm{~mm}$, with a homogeneous size-distribution of the width $1-$ $\mathscr{A}=1-\langle a\rangle^{2} /\left\langle a^{2}\right\rangle=0.18922$ (with $a_{\min } / a_{\max }=$ $1 / 2)$. The size of particles is chosen so as to mimic the original experiments by Ref. (Fenistein et al. 2004). The stiffness constant of the particles is $k=100 \mathrm{Nm}^{-2}$. The rolling and torsion friction are inactive. The normal and tangential viscosities are $\gamma_{n}=0.002 \mathrm{~kg} \mathrm{~s}^{-1}$ and $\gamma_{t} / \gamma_{n}=1 / 4$. In order to study the influence of contact friction, we analyzed the system for the following set of friction coefficients:

$\mu_{\mathrm{p}} \in[0,0.005,0.01,0.02,0.05,0.1,0.2,0.5,1.0]$

The initial state is prepared without any friction between particles, and different values of $\mu_{\mathrm{p}}$ are activated at the onset of shearing. Details about initial preparation of the packing are reported in (Luding 2008b, Luding 2008a).

\subsection{Split-bottom ring shear cell}

Figure 1 is a sketch of our numerical setup; the geometry of the system is described in detail in Ref. (Fenistein et al. 2004)). In this figure, the inner, split, and outer radii are given by $R_{i}, R_{s}$, and $R_{o}$, respectively, where the concentric cylinders rotate relative to each other around the symmetry axis (the dot-dashed line). The ring shaped split at the bottom separates the moving and static parts of the system, where a part of the bottom and the outer cylinder rotate at the same rate. The system is filled with $N \approx 3.7 \times 10^{4}$ spherical particles with density $\rho=$ $2000 \mathrm{~kg} / \mathrm{m}^{3}=2 \mathrm{~g} / \mathrm{cm}^{3}$ up to height $H$. The cylindrical walls and the bottom are roughened due to some (about $3 \%$ of the total number) attached/glued particles (Luding 2008b, Luding 2008a).

When there is a relative motion at the split, a shear band propagates from split position $R_{S}$ upwards and inwards and remains far away from cylinder-walls and bottom in most cases.

Since we are interested in the quasi-static regime, the rotation rate of outer cylinder is chosen to be $f_{o}=$ $0.01 \mathrm{~s}^{-1}$, such that the inertial number $I \ll 1$.

\subsection{Averaging and micro-macro proce- dure}

Translational invariance is assumed in the tangential $\phi$-direction, the averaging is performed over toroidal volumes over many snapshots in time. leading to field $Q(r, z)$ as function of the radial and vertical positions. The averaging procedure has been explained in detail for 2D systems in (Lätzel et al. 2000), and three dimensional systems in (Luding 2008b, Luding 2008a), and will not be discussed here. The simulation runs for more than $50 \mathrm{~s}$. For the spatial and time averaging, only large times are taken into account, disregarding the transient behavior at the onset of shear.

From the simulations, one can calculate the stress tensor as

$\sigma_{i j}=\frac{1}{V}\left[\sum_{p \in V} m^{p}\left(v_{i}^{p}\right)\left(v_{j}^{p}\right)-\sum_{c \in V} r_{i}^{c} f_{j}^{c}\right]$

with $p$ particles, mass $m^{p}$, velocity $v^{p}$, force $f^{c}$ and branch vector $r^{c}$. The velocity $v^{p}$ is relative to the mean streaming velocity inside the averaging volume $V$. The first term is the sum of kinetic energy fluctuations, and the second involves the dyadic product of contact-force with the contactbranch vector. The contribution of the kinetic energy stress to the total shear stress $\sigma$ is much smaller compared to the contact stress (for the rotation rate we are interested in). Hence, we ignore the dynamical stress and concentrate on the contact stress.

The quantity which describes the local network of contacts in a granular material is the fabric tensor (Luding 2005), defined as

$F_{i j}=\frac{1}{V} \sum_{p \in V} V^{p} \sum_{c \in p} n_{i}^{c} n_{j}^{c}$ 
where $V^{p}$ is the particle volume which lies inside the averaging volume $V, n^{c}$ is the normal unit branch-vector pointing from center of particle $p$ to contact $c$.

For both stress and fabric tensors, we can calculate the eigenvalues and define the volumetric part $T_{\mathrm{v}}=\left(T_{1}+T_{2}+T_{3}\right) / 3$ (pressure $p$ and $F_{\mathrm{v}}$ for stress and fabric respectively) and deviatoric component as $T_{\mathrm{dev}}=\sqrt{\left(\left(T_{1}-T_{2}\right)^{2}+\left(T_{2}-T_{3}\right)^{2}+\left(T_{3}-T_{1}\right)^{2}\right) / 6}$ ( $\sigma_{\mathrm{dev}}$ and $F_{\mathrm{dev}}$ for stress and fabric respectively).

The pressure is the isotropic stress, while $\sigma_{\mathrm{dev}}$ quantifies the normal stress difference and the shear stress. The volumetric fabric $F_{\mathrm{v}}$ represents the contact number density, while the deviatoric fabric $F_{\mathrm{dev}}$ quantifies anisotropy of the contact network.

Since we are interested in the flow behavior, the data only inside the flowing part of the system i.e., inside the shear band is analyzed.

\section{Results}

For a given confining stress (and preparation history), the material can only resist shear up to certain limit of (shear) stress, called as yield stress beyond which it fails (Schwedes 2003). When yield points $\left(p^{(y)}, \sigma^{(y)}\right.$ dev $)$ are collected in the $\sigma_{\mathrm{dev}}-p$-plane, a yield locus can be identified that fully describes the failure behavior of the material. In addition, when the material is sheared continuously for a long time, it reaches a state which is characterized by a steady state stress, i.e. the stress needed to keep the material in motion, $\left(p, \sigma_{\mathrm{dev}}\right)$, also referred to as the critical state. For simple granular materials, the termination locus can be predicted from a Coulomb type criterion as a straight line whose slope is macroscopic friction coefficient $\mu_{\text {macro }}=\left(\sigma_{\mathrm{dev}}\right) / p$.

In the following sections, we study the effect of the particle friction coefficient on the macroscopic behavior of the material and the probability density functions (PDFs) for systems in steady state shear.

\subsection{Effect of particle friction on macro- scopic behavior}

Our previous study has reported (Singh et al. 2013) the effect of particle friction on the steady state shear strength and contact anisotropy. We have shown that volumetric fabric $F_{\mathrm{v}}$ decreases monotonically with increasing $\mu_{\mathrm{p}}$. This was explained based on the fact that for higher contact friction, a single particle needs less contacts to be in mechanical equilibrium. On the other hand, we have found that $F_{\mathrm{dev}}$ remains almost constant against pressure, while its value increases with contact friction until a saturation value is reached. A similar behavior was observed for $\mu_{\text {macro }}$ vs contact friction $\mu_{\mathrm{p}}$. Here, we go further and look in detail at the influence of friction on the steady state behavior, focusing on the strong/weak force subnetworks and their contribution to both stress and fabric deviators. We split the contacts into weak and strong subnetworks, characterized by $f^{*}(P)=f(P) /\langle f(P)\rangle$ smaller or larger than 1 , respectively.

Fig. 2 illustrates the relative contributions of subnetworks with $f^{*}<1$ and $f^{*}>1$ to the deviatoric stress $\sigma_{\mathrm{dev}}$ and deviatoric fabric $F_{\mathrm{dev}}$, together with the overall network. Fig. 2(a) clearly shows that the contribution of the weak subnetwork to $\sigma_{\mathrm{dev}}$ is negligible. In Fig. 2(b) we plot the contribution of weak and strong subnetworks to $F_{\mathrm{dev}}$. In agreement with previous studies (Radjai et al. 1998, Thornton and Antony 2000), a much stronger fabric anisotropy characterizes the strong subnetwork, while the weak subnetwork is mostly isotropic. It is noteworthy that $F_{\text {dev }}$ for both subnetworks is positive because of the definition of deviator, but we expect the main eigenvectors of strong and weak subnetworks to have orthogonal directions.

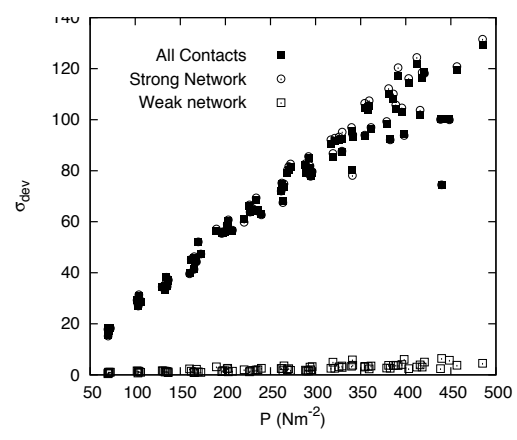

(a)

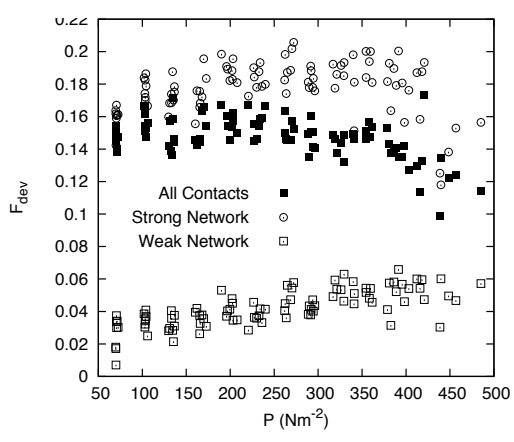

(b)

Figure 2: Contribution of weak, and strong subnetworks to (a) $\sigma_{\text {dev }}$ and (b) $F_{\text {dev }}$ plotted against pressure $P$.

Fig. 3(a) displays the normalized shear stress $s_{D}=$ $\sigma_{\mathrm{dev}} / P$ as a function of $\mu_{\mathrm{p}}$ for the two subnetworks and the overall system. We observe that for the whole range of particle friction $\mu_{\mathrm{p}}$, the strong subnetwork carries almost all $s_{D}$. System with $\mu_{\mathrm{p}}=0$ has finite $s_{D}$ due to interlocking of contacts (Singh et al. 2013). The whole network and the strong subnetwork show similar variation in $s_{D}$ as a function of $\mu_{\mathrm{p}}$, i.e. an initial increase and then saturation for high $\mu_{\mathrm{p}}$.

Fig. 3(b), displays $F_{\mathrm{dev}}$ as a function of $\mu_{\mathrm{p}}$ for the strong and weak subnetworks and the complete network. We observe that the strong subnetwork car- 
ries higher anisotropy than the overall network. It is interesting to note that, in a similar manner $F_{\mathrm{dev}}$ also starts with a non-zero value for zero $\mu_{\mathrm{p}}$ i.e. the contact network for frictionless particles is also anisotropic. It increases with initial increase in $\mu_{\mathrm{p}}$ until it saturates for high $\mu_{\mathrm{p}} \geq 0.3$.

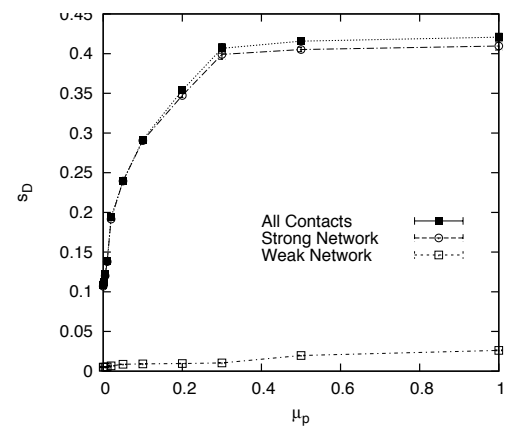

(a)

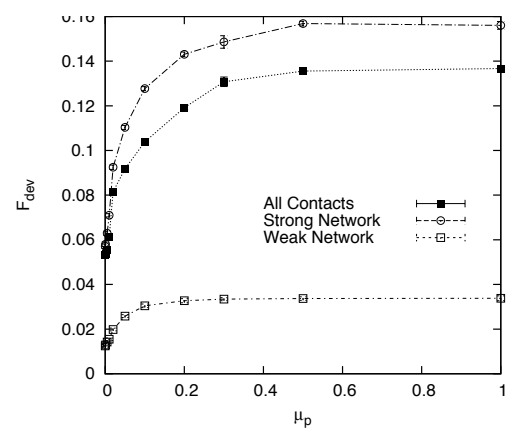

(b)

Figure 3: Contribution of weak, and strong subnetworks to (a) $s_{D}=\sigma_{\mathrm{dev}} / P$ and (b) $F_{\mathrm{dev}}$ plotted against contact coefficient of friction $\mu_{\mathrm{p}}$.

\subsection{Force Distribution}

To better understand the macroscopic observations in the previous section, we focus on its micromechanical origin and we extend our analysis by looking not only at the two strong/weak subnetworks, but also at the full probability distribution function of forces, which carries the information about the inhomogeneities of force network.

Pioneering work of Liu et al. (Liu et al. 1995) using carbon paper technique indicated that the number of contacts carrying a given force decreased as the magnitude of the force increased. From the work of (Silbert 2010), it was shown that the probability distribution is also sensitive to preparation history, packing disorder and various particle properties like contact friction.

Normal forces The probability distributions for normalized normal forces $f_{n}^{*}=f_{n} /\left\langle f_{n}\right\rangle$ are shown in Fig. 4 for different values of $\mu_{\mathrm{p}}$ (for the sake of clarity we show only 4 values of $\mu_{\mathrm{p}}$ ). We observe a very similar behavior for $P\left(f_{n}^{*}\right)$ in the range of strong forces $f_{n}^{*}>1$, independent of friction. However, the contact friction affects the distribution of weak forces $f^{*}<1$, as for $\mu_{\mathrm{p}}=0.0$ and 0.01 , the distribution bends down as $f_{n}^{*} \rightarrow 0$, but does not tend to zero, while an opposite is observed for high $\mu_{\mathrm{p}}$. The difference in shape of $P\left(f_{n}^{*}\right)$ for $f_{n}^{*}<1$ was also observed in Refs. (Silbert 2010, Azéma et al. 2009). This shows that the fraction of contacts carrying weak forces increases with increasing $\mu_{\mathrm{p}}$, even though the total number of contacts decrease. The inset of the figure also shows that with increasing friction, the tail of distribution in Fig. 4 gets longer (from $\mu_{\mathrm{p}}=0.0$ to 1.0 ), stating that the inhomogeneity in the contact network increases with friction.

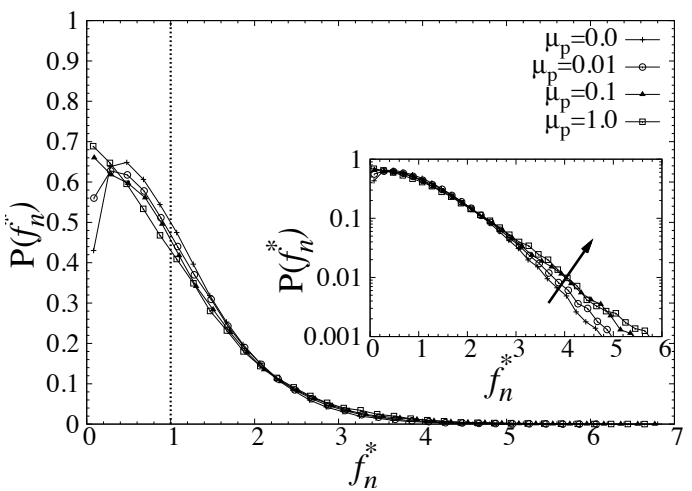

Figure 4: Probability density functions of normalized normal forces. The inset shows the same on a log-linear scale.

Furthermore, we look at $P\left(f_{n}^{*}\right)$ along the principal eigen-directions of the local strain rate tensor of the overall network (Duran et al. 2010). Fig. 5 displays $P\left(f_{n}^{*}\right)$ of forces for contacts aligned with the compressive directions of local strain rate tensor normalized by the mean force of overall network. We observe that the tail of the distribution gets longer, with increasing $\mu_{\mathrm{p}}$, i.e. it leads to inhomogeneity in the forces along the compressive direction. For all range of particle friction, the tensile subnetwork (not shown here) always stays more homogeneous than the compressive. This implies that friction mainly affects the force distribution along the compressive direction.

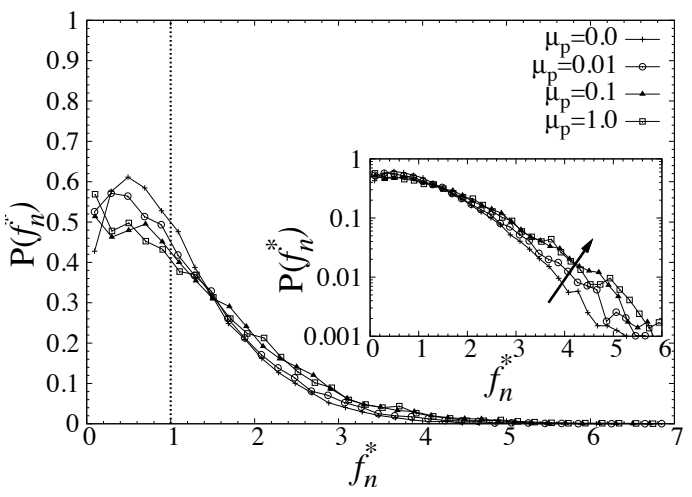

Figure 5: Probability density functions of normal forces along the compressive direction normalized by overall normal forces. The inset shows the same on a log-linear scale. 
Tangential forces Fig. 6 shows the distribution of the tangential forces, that behaves very similarly to $P\left(f_{n}^{*}\right)$ for normal forces, with a friction dependent head for $f^{*}<1$ and no dependence for strong forces.

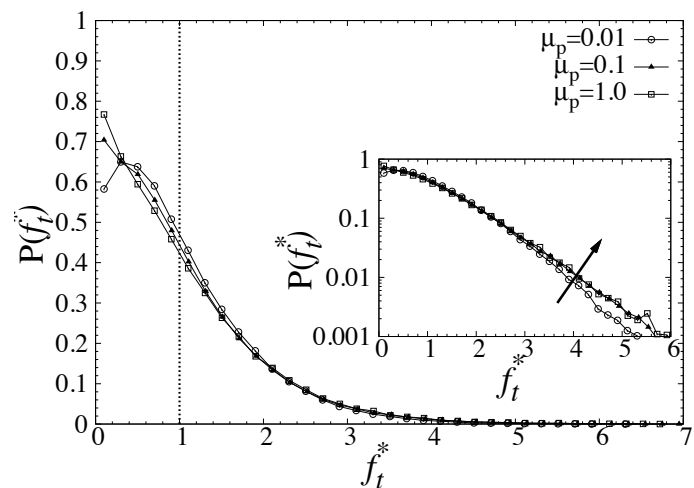

Figure 6: Probability density functions of normalized tangential forces. The inset shows the same on a log-linear scale.

For the sake of completeness, we also analyze the probability distribution of tangential forces along the compressive and tensile directions, as done previously. Surprisingly, the behavior is now different between normal and tangential components, as the latter does not show any trend with friction in the compressive direction ( Fig. 8). This implies that friction does not affect the tangential forces along the eigenvectors, but only the whole distribution of forces, displaying a different mechanism between normal and tangential forces.

\subsection{Anisotropy of force networks}

Fig. 7 shows the $P\left(f_{n}^{*}\right)$ for normal forces along the eigen-directions for different values of $\mu_{\mathrm{p}} . P\left(f_{n}^{*}\right)$ of overall network lies in between the $P\left(f_{n}^{*}\right)$ of forces in compressive and tensile directions. For all values of $\mu_{\mathrm{p}}$, we observe that the $P\left(f_{n}^{*}\right)$ of weak forces $\left(f_{n}^{*}<1\right)$ along the tensile direction is higher compared to that in the compressive direction, which is intuitive as the majority of contacts will have small forces in the tensile direction. However, as the value of force increases, $P\left(f_{n}^{*}>1\right)$ the probability along the compressive directions overcomes the one in tensile direction. We also observe that with increasing $\mu_{\mathrm{p}}$, the difference between the distributions along compressive and tensile directions increases, indicating that the anisotropy in the force network (compressive forces) increases.

Fig. 8 displays the same comparison for tangential forces. Again, no clear trend of $P\left(f_{t}^{*}\right)$ can be inferred from the figure (besides the fluctuations), as a confirmation that friction does not affect the behavior of the tangential component along the eigenvectors.

\section{Discussion}

The effect of contact friction on both macroscopic and microscopic properties of a granular assembly have been studied by means of the discrete element method (DEM), in search of the connection between them. While analyzing the contact network, split in weak and strong subnetworks, we observe that the latter carries the majority of the stress and fabric anisotropy, having values increasing and then saturating with friction.

Looking at the microscale, we find that friction affects the probability distribution of normal and tangential forces in a very similar fashion: the increase of contact friction leads to an increase of the heterogeneity (width of the PDF) in both the force subnetworks. However, when the focus moves on the probability distributions along compressive and tensile directions, a systematic increase in anisotropy (difference between the mean values along the two directions) is seen with increasing friction, but only for normal forces. Surprisingly, no such anisotropy is present for tangential forces. Thus, friction influences the behavior of the force network in both its components moving from a Gaussian-like bellshaped distribution with a peak around the mean, for weak friction to an exponential type with continuously decreasing probability with force for stronger friction. While this effect clearly shows up along the eigen-direction for normal forces indicating that friction strengthens the force chains and releases the weak network, a different mechanism acts for the tangential forces, which deserves further investigation in the future.

As a final conclusion, we show that the shear strength of strong force network gets affected by particle friction because of change in the fabric $F$, while in the case of weak force network both fabric and force distributions (PDFs) are affected. This conclusion is consistent with a recent study (Azéma \& Radjaï 2014).

\section{Acknowledgment}

We thank K. Saitoh, O. I. Imole, N. Kumar and T. Weinhart for helpful discussions. Financial support through the "Jamming and Rheology" project of NWO-FOM is acknolwdged.

\section{REFERENCES}

Azéma, E. \& F. Radjaï (2014). Internal structure of inertial granular flows. Physical Review Letters 112(7), 078001.

Azéma, E., F. Radjai, \& G. Saussine (2009). Quasistatic rheology, force transmission and fabric properties of a packing of irregular polyhedral particles. Mechanics of Materials 41(6), 729 - 741. Advances in the Dynamics of Granular Materials.

Cundall, P. A. (1971). A computer model for simulating progressive, large-scale movements in blocky rock systems. In Proc. Symp. Int. Rock Mech., Volume 2, Nancy.

Duran, O., N. P. Kruyt, \& S. Luding (2010). Micromechanical analysis of deformation characteristics of three-dimensional granular materials. Int. J. of Solids and Structures 47, 2234-2245.

Fenistein, D., J. W. van de Meent, \& M. van Hecke (2004). Universal and wide shear zones in granular 


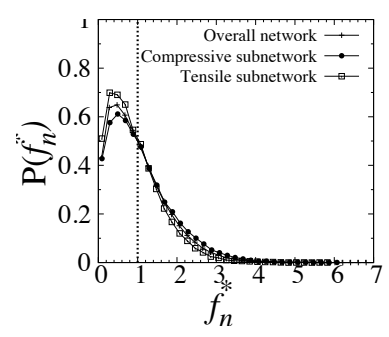

(a)

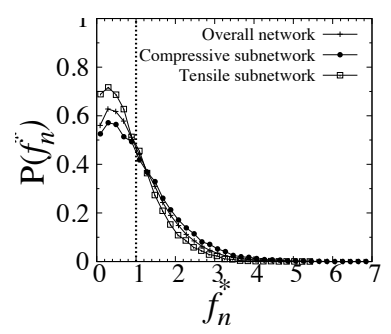

(b)

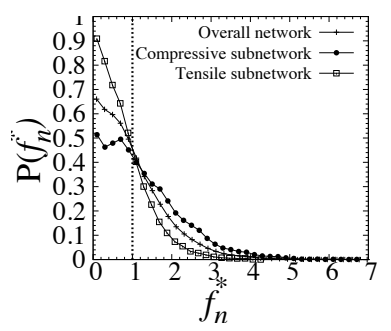

(c)

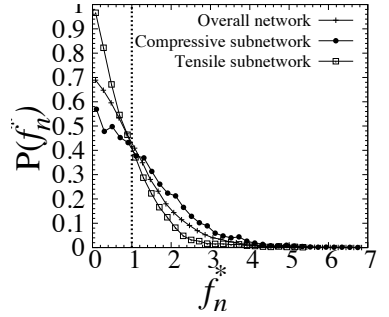

(d)

Figure 7: Probability density functions of normal forces along the compressive, tensile directions, and overall network in red, blue and green colors for $\mu_{\mathrm{p}}=$ (a) 0 , (b) 0.01 , (c) 0.1 , and (d) 1.0 in linear scale. The forces are normalized by the mean normal force of the overall network.

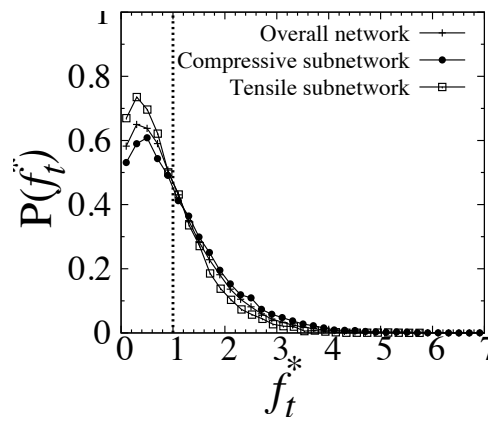

(a)

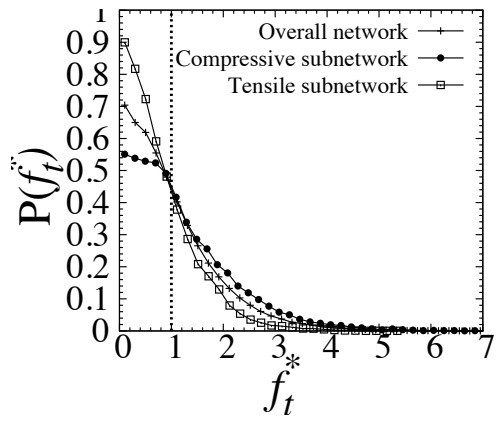

(b)

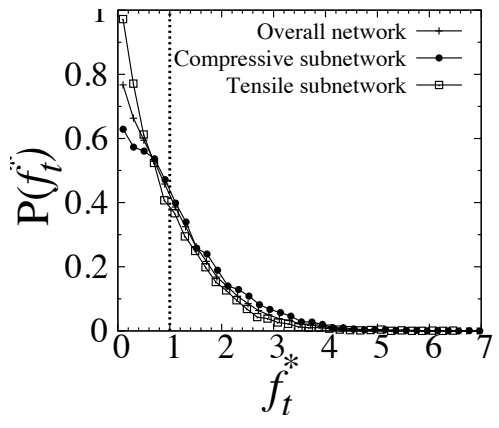

(c)

Figure 8: Probability density functions of tangential forces along the compressive, tensile directions, and overall network in red, blue and green colors for $\mu_{\mathrm{p}}=$ (a) 0.01 , (b) 0.1 , and (c) 1.0 in linear scale. The forces are normalized by the mean tangential force of the overall network.

bulk flow. Phys. Rev. Lett. 92, 094301. e-print condmat/0310409.

Jaeger, H. M., S. R. Nagel, \& R. P. Behringer (1996). Granular solids, liquids, and gases. Rev. Mod. Phys. 68(4), 1259-1273.

Lätzel, M., S. Luding, \& H. J. Herrmann (2000). Macroscopic material properties from quasi-static, microscopic simulations of a two-dimensional shearcell. Granular Matter 2(3), 123-135. e-print condmat/0003180.

Liu, C., S. R. Nagel, D. A. Schecter, S. N. Coppersmith, S. Majumdar, O. Narayan, \& T. A. Witten (1995). Force fluctuations in bead packs. Science 269, 513.

Luding, S. (2005). Anisotropy in cohesive, frictional granular media. Journal of Physics Condensed Matter 17(24), S2623-S2640.

Luding, S. (2008a). Constitutive relations for the shear band evolution in granular matter under large strain. Particuology 6(6), 501-505.

Luding, S. (2008b). The effect of friction on wide shear bands. Particulate Science and Technology 26(1), 3342.

Luding, S. \& F. Alonso-Marroquín (2011). The criticalstate yield stress (termination locus) of adhesive powders from a single numerical experiment. Granular Matter 13, 109-119.

Radjai, F., D. E. Wolf, M. Jean, \& J.-J. Moreau (1998). Bimodal character of stress transmission in granular packings. Phys. Rev. Lett. 80(1), 61-64.

Schwedes, J. (2003). Review on testers for measuring flow properties of bulk solids. Granular Matter 5(1), $1-45$.

Silbert, L. E. (2010). Jamming of frictional spheres and random loose packing. Soft Matter 6, 2918-2924.

Singh, A., V. Magnanimo, \& S. Luding (2013). Effect of friction and cohesion on anisotropy in quasi-static granular materials under shear. AIP Conference Proceedings 1542(1).

Thornton, C. \& S. J. Antony (2000). Quasi-static deformation of a soft particle system. Pow. Tech. 109(1-3), 179-191. 